\title{
How to Perform Curative Laparoscopic Hepatectomy for Intraoperatively Unidentified Hepatocellular Carcinoma
}

\author{
MASASHI TSUNEMATSU, SHINJI ONDA, MITSURU YANAGAKI, NORIMITSU OKUI, \\ TADASHI UWAGAWA, JUNGO YASUDA, KENEI FURUKAWA, \\ KOICHIRO HARUKI, TAKESHI GOCHO, YOICHI TOYAMA and TORU IKEGAMI \\ Division of Hepatobiliary Pancreatic Surgery, Department of Surgery, \\ The Jikei University School of Medicine, Tokyo, Japan
}

\begin{abstract}
Background/Aim: Detection of hepatocellular carcinoma using intraoperative ultrasonography (IOUS) is indispensable for successful laparoscopic hepatectomy (LH). This study was performed to evaluate patients with intraoperatively unidentified tumours undergoing LH. Patients and Methods: Seven patients who underwent LH for hepatocellular carcinoma and whose tumours were not detected using IOUS were included in this study. Clinical features, preoperative imaging, intraoperative imaging, surgical procedures, and pathological findings were evaluated. Results: Using gadolinium ethoxybenzyl diethylenetriamine pentaacetic acid-enhanced magnetic resonance imaging, all the tumours were enhanced in the arterial phase and rapidly washed out, becoming hypointense to the remainder of the liver. All tumours except one were $<2 \mathrm{~cm}$ in size. Severe liver fibrosis was observed in all cases. Tumours that were invisible on preoperative ultrasonography also could not be detected using IOUS or indocyanine green fluorescence imaging. Five patients underwent hepatectomy based on anatomical landmarks and achieved curative resection, whereas curative resection failed in two patients. Conclusion: When tumours cannot be identified by IOUS, LH based on anatomical landmarks should be preferred. Importantly, invisible tumours on preoperative ultrasonography may not be identified intraoperatively during $\mathrm{LH}$.
\end{abstract}

Laparoscopic hepatectomy (LH) has recently been recognized as

This article is freely accessible online.

Correspondence to: Shinji Onda, Division of Hepatobiliary Pancreatic Surgery, Department of Surgery, The Jikei University School of Medicine, 3-25-8, Nishi-Shinbashi, Minato-ku, Tokyo 105-8461, Japan. Tel: +81 334331111 ext. 3401, Fax: +81 354724140, e-mail: s-onda@jikei.ac.jp

Key Words: Hepatocellular carcinoma, laparoscopic hepatectomy, intraoperative ultrasonography. a treatment option for hepatocellular carcinoma (HCC) by advances in instruments and surgical techniques (1). Preoperative and intraoperative identification of the tumour is important for patients undergoing LH. Dynamic computed tomography (CT) and gadolinium ethoxybenzyl diethylenetriamine pentaacetic acid-enhanced magnetic resonance imaging (Gd-EOB-MRI) are widely used for preoperative assessment of HCC (2), while intraoperative ultrasonography (IOUS) of the liver is a mandatory step for detecting tumours (3).

Although laparoscopic IOUS is as reliable as open IOUS in detecting liver tumours and planning liver resection (4), the nodularity of chronic liver disease and adhesions make the localization and identification of tumours difficult using laparoscopic IOUS. We sometimes encounter cases in which tumours are not detected intraoperatively.

This study was performed to evaluate patients who underwent LH for invisible HCC and establish effective strategies for performing curative LH in these patients.

\section{Patients and Methods}

Patients. From July 2017 to April 2021, 75 patients underwent LH for HCC at The Jikei University Hospital, Tokyo, Japan. Of these, seven patients with intraoperative invisible hepatic tumours were included in the study. We retrospectively reviewed a prospectively maintained patient database. The following patient information was obtained from the prospective database: sex, age, serum $\alpha-$ foetoprotein level, serum des-gamma-carboxy prothrombin level, diagnosis of underlying chronic liver disease, details of surgical procedure, and pathological findings. The laboratory measurements were routinely obtained immediately before surgery. This research was approved by the institutional ethics committee of The Jikei University School of Medicine (27-177(8062)) and conformed to the provisions of the Declaration of Helsinki. Continuous variables are expressed as mean \pm standard deviation.

Preoperative evaluation of tumours. All patients routinely underwent preoperative ultrasonography (POUS) (Aplio 300; CANON Medical Systems Corporation, Tochigi, Japan) as well as multiphase contrastenhanced CT scans and Gd-EOB-MRI unless they had allergies to 
contrast media or severe renal failure. A skilled medical laboratory technician and a surgeon performed POUS to confirm the tumour location and its relationship with the adjacent hepatic vasculature. Dynamic CT imaging was performed using a 64-slice multidetector row CT scanner (SOMATOM Definition AS+; Siemens Healthineers, Erlangen, Germany) after administration of a rapid bolus injection of iodinated contrast agent at a rate of 2.5 to $4.0 \mathrm{ml} / \mathrm{s}$ using a power injector. We obtained the arterial, portal, and venous phases at 30 , 60 , and $180 \mathrm{~s}$, respectively. The slice thickness was $1 \mathrm{~mm}$, and the reconstruction interval was $5 \mathrm{~mm}$.

All hepatic MRI scans were performed on a 3T MRI scanner (MAGNETOM Skyra; Siemens Healthineers). For Gd-EOB-MRI, the recommended full dose of gadoxetic acid (EOB Primovist; Bayer HealthCare, Osaka, Japan) based on body weight $(0.1$ $\mathrm{ml} / \mathrm{kg}$ ) was intravenously injected. A multiphase dynamic study including the arterial, portal, and late phases was carried out. T1weighted turbo field-echo sequences were acquired in the hepatobiliary phase, 10 to $15 \mathrm{~min}$ after the injection of gadoxetic acid. The depth of the tumour from the liver surface was measured in the hepatobiliary phase. Preoperative dynamic CT and Gd-EOBMRI were performed within 1 month before surgery and retrospectively reviewed by two surgeons (M.T. and S.O.) and two radiologists specializing in $\mathrm{HCC}$.

Surgical procedure. For lesions located in the posterior segment, patients were placed in the semi-left lateral position, whereas for lesions located at sites other than the posterior segment, patients were placed in the supine position. The first port was inserted using an open method. After establishment of pneumoperitoneum, the abdominal cavity was deftly observed using a flexible laparoscope. If intraperitoneal adhesions obscured the operative field, the adhesions were carefully dissected using a vessel-sealing device (LigaSure $^{\mathrm{TM}}$ Maryland Jaw device; Medtronic Inc., Dublin, Ireland). Five ports were inserted under direct vision depending on the location of the tumour. An additional 5-mm intercostal transthoracic balloon port (Kii Balloon Access Systems ${ }^{\mathrm{TM}}$; Applied Medical Inc., Rancho Santa Margarita, CA, USA) was inserted as necessary. IOUS (Aplio 300; CANON Medical Systems Corporation, Tochigi, Japan) was routinely performed to confirm the tumour location and its relationship with the adjacent hepatic vasculature. Intraoperative indocyanine green (ICG) fluorescence imaging (VISERA ELITE II; Olympus, Tokyo, Japan) was routinely performed to detect hepatic tumours. In this technique, ICG $(0.5 \mathrm{mg} / \mathrm{kg}$ body weight $)$ was administered intravenously 1 or 2 days before surgery. The Pringle manoeuvre was performed, in which cycles of 15 minutes of clamping were alternated with 5 minutes of reperfusion. The hepatic parenchyma was divided using a cavitron ultrasonic surgical aspirator (CUSA; Integra LifeSciences Inc., Princeton, NJ, USA), and each of the encountered vessels was divided using clips and vessel-sealing devices. The resected specimen was retrieved using a reinforced laparoscopic retrieval bag.

\section{Results}

The patients' clinicopathological characteristics are shown in Table I. The mean age of the seven patients was $65.4 \pm 6.2$ years. The mean diameter of the tumours was $14 \pm 5 \mathrm{~mm}$. The locations of the tumours were segment $2(n=3)$, segment 4 $(n=1)$, segment $5(n=1)$, segment $6(n=1)$, and segment $7(n=1)$.
The surgical procedures included segmentectomy $(n=2)$ and partial resection $(n=4)$. Repeat hepatectomy was performed in four patients. The pathological diagnoses were HCC $(n=4)$, a dysplastic nodule $(n=1)$, and none of the tumour $(n=1)$.

On Gd-EOB-MRI, all the tumours were enhanced in the arterial phase and rapidly washed out, becoming hypointense to the remainder of the liver (Table II). Only one tumour was detected using POUS, and the invisible tumours on POUS could not be detected using IOUS. No tumour was detected using ICG fluorescence imaging; this reflected the progression of liver fibrosis.

Hepatectomies based on anatomical landmarks were performed except in one patient who did not undergo hepatectomy. One patient underwent failed curative resection because of misidentification of the anatomical landmark (Patient \#4).

Patient \#1 was a 61-year-old man with a history of repeat transcatheter arterial chemoembolization for HCC who developed recurrent HCC in segments 1 and 4 (Figure 1A). On Gd-EOB-MRI, the tumour in segment 4 was enhanced in the arterial phase and rapidly washed out, becoming hypointense to the remainder of the liver (Figure 1B). The tumour in segment 4 was not detected by either POUS or IOUS. Because it was invisible on normal light and ICG fluorescence imaging (Figure 1C and D), we performed laparoscopic partial hepatectomy for HCC in only segment 1 . We planned to carefully follow up the untreated tumour in segment 4.

Patient \#5 was a 59-year-old man with a history of open right lobectomy for HCC who developed recurrent HCC in segment 2. On dynamic CT, the tumour was vividly enhanced during the late arterial phase and then washed out rapidly (Figure 2A). On Gd-EOB-MRI, it was also enhanced in the arterial phase and rapidly washed out, becoming hypointense to the remainder of the liver (Figure 2B). It was not detected using POUS. The tumour was invisible on both IOUS and ICG fluorescence imaging, and we performed G2-oriented partial hepatectomy (as for Patient \#4). Postoperative CT revealed that $\mathrm{G} 2$ had been misrecognized as G3 (Figure 2C). Therefore, we performed subsegmentectomy of segment 2, and curative resection was achieved 5 months after the first repeat hepatectomy (Figure 2D).

The other patients underwent hepatectomy based on anatomical landmarks: hepatectomy along the cut surface of previous right lobectomy (Patient \#2), G3-oriented partial hepatectomy (Patient \#3), subsegmentectomy of segment 2 (Patient \#4), and hepatectomy along with a nodule on the surface of the liver.

\section{Discussion}

In the present study, tumours that were invisible on POUS could not be detected using IOUS during LH for HCC, and no tumour was detected using ICG fluorescence imaging. 
Table I. Patient clinicopathological characteristics.

\begin{tabular}{|c|c|c|c|c|c|c|c|c|c|c|c|}
\hline \multirow[t]{2}{*}{ Case } & \multirow{2}{*}{$\begin{array}{l}\text { Age } \\
\text { years }\end{array}$} & \multirow[t]{2}{*}{ Gender } & \multicolumn{4}{|c|}{ Preoperative evaluation of tumor } & \multirow{2}{*}{$\begin{array}{l}\text { Laparoscopic } \\
\text { hepatectomy }\end{array}$} & \multicolumn{2}{|c|}{ Pathological findings } & \multicolumn{2}{|c|}{ Underlying liver } \\
\hline & & & Location & $\begin{array}{l}\text { Maximum } \\
\text { size } \\
\mathrm{mm}\end{array}$ & $\begin{array}{l}\text { Depth from } \\
\text { liver surface } \\
\text { mm }\end{array}$ & $\begin{array}{c}\text { Repeat } \\
\text { hepatectomy }\end{array}$ & & Diagnosis & Differentiation & $\begin{array}{l}\text { Chronic } \\
\text { liver } \\
\text { disease }\end{array}$ & $\begin{array}{c}\text { New } \\
\text { Inuyama } \\
\text { classification }\end{array}$ \\
\hline$\# 1$ & 63 & M & $\mathrm{S} 4$ & 11 & 0 & No & No resection & & & $\mathrm{HCV}$ & F3 \\
\hline$\# 2$ & 69 & $\mathrm{~F}$ & S5 & 12 & 2.5 & Yes & Partial & $\mathrm{HCC}$ & Well & $\mathrm{HCV}$ & $\mathrm{F} 4$ \\
\hline \#3 & 63 & M & S7 & 24 & 2.4 & No & Partial & $\mathrm{HCC}$ & Well & NASH & F3 \\
\hline \#4 & 59 & M & $\mathrm{S} 2$ & 15 & 0 & Yes & Partial & None & & ALD & F3 \\
\hline$\# 5$ & 59 & M & $\mathrm{S} 2$ & 10 & 0 & Yes & Segmentectomy & $\mathrm{DN}$ & & ALD & F3 \\
\hline \#6 & 68 & M & $\mathrm{S} 2$ & 11 & 16.4 & Yes & Segmentectomy & $\mathrm{HCC}$ & Well & NASH & F3 \\
\hline$\# 7$ & 76 & $\mathrm{~F}$ & S6 & 14 & 0 & No & Partial & $\mathrm{HCC}$ & Well & $\mathrm{HCV}$ & $\mathrm{F} 4$ \\
\hline
\end{tabular}

ALD: Alcoholic liver disease; DN: dysplastic nodule; F: female; HCC: hepatocellular carcinoma; HCV: hepatitis C virus; M: male; NASH: nonalcoholic steatohepatitis; S: segment.

Table II. Tumour identification using each modality.

\begin{tabular}{|c|c|c|c|c|c|c|c|c|}
\hline Case & $\begin{array}{l}\text { Classical HCC } \\
\text { on dynamic CT }\end{array}$ & $\begin{array}{c}\text { Positive on } \\
\text { DWI MRI }\end{array}$ & $\begin{array}{c}\text { Early } \\
\text { enhancement } \\
\text { on } \\
\text { EOB-MRI }\end{array}$ & $\begin{array}{l}\text { Wash-out on } \\
\text { hepatobiliary } \\
\text { phase of } \\
\text { EOB-MRI }\end{array}$ & $\begin{array}{l}\text { Detectable } \\
\text { on } \\
\text { preoperative } \\
\text { US }\end{array}$ & $\begin{array}{c}\text { Detectable } \\
\text { on } \\
\text { intraoperative } \\
\text { US }\end{array}$ & $\begin{array}{c}\text { Positive on } \\
\text { intraoperative } \\
\text { ICG-fluorescence } \\
\text { imaging }\end{array}$ & $\begin{array}{l}\text { Curative } \\
\text { resection }\end{array}$ \\
\hline \#1 & & Yes & Yes & Yes & No & No & No & No \\
\hline \#2 & Yes & Yes & Yes & Yes & No & No & No & Yes \\
\hline \#3 & Yes & Yes & Yes & Yes & No & No & No & Yes \\
\hline \#4 & Yes & No & Yes & Yes & No & No & No & No \\
\hline$\# 5$ & Yes & No & Yes & Yes & No & No & No & Yes \\
\hline$\# 6$ & Yes & Yes & Yes & Yes & Yes & No & No & Yes \\
\hline \#7 & & No & Yes & Yes & No & No & No & Yes \\
\hline
\end{tabular}

CT: Computed tomography; DWI: diffusion-weighted imaging; Gd-EOB: gadolinium ethoxybenzyl diethylenetriamine pentaacetic acid-enhanced; HCC: hepatocellular carcinoma; ICG: indocyanine green; MRI: magnetic resonance imaging; US: ultrasonography.

Hepatectomy based on anatomical landmarks was feasible for patients whose tumours were not detected intraoperatively.

We believe that there are three main reasons why tumours cannot be identified intraoperatively: tumour-related factors, underlying liver conditions, and technical factors. Tumourrelated factors include the size, location, and differentiation of the tumours. In all but one patient in the present study, the size of the tumours was $<2 \mathrm{~cm}$. Previous studies have shown that small HCCs of $<2 \mathrm{~cm}$ are often poorly visible using ultrasonography $(5,6)$. In addition, differentiation from cirrhotic nodules on the liver surface remains difficult with IOUS because of focal length mismatch (7). Underlying liver conditions include liver fibrosis and anatomical alteration of the liver caused by regeneration due to cirrhosis or previous hepatectomy. Liver fibrosis was severe in all cases. With respect to technical factors, laparoscopic IOUS is a reliable tool similar to open IOUS, and technical factors depend on the operability of laparoscopic IOUS (8). In particular, during repeat LH, IOUS is difficult because of loss of anatomical landmarks and anatomical alterations of the liver caused by regeneration after the previous hepatectomy. Four $(57 \%)$ patients who underwent repeat LH were enrolled in this study. The tumours that were not detected on POUS remained undetected on IOUS. Intraoperative fluorescence imaging following preoperative intravenous injection of ICG is an adjunct to IOUS (9). Well-differentiated HCCs are detected as uniformly and highly fluorescing lesions (10). However, ICG fluorescence imaging can detect tumours that are located only up to $8 \mathrm{~mm}$ from the hepatic surface because of the limitation of tissue penetration of fluorescence signals (11). In addition, because ICG fluorescence imaging reveals fluorescence in regenerative nodules as well as HCCs, identification of the target tumour becomes difficult under the cirrhosis milieu (12). Despite this limitation in 

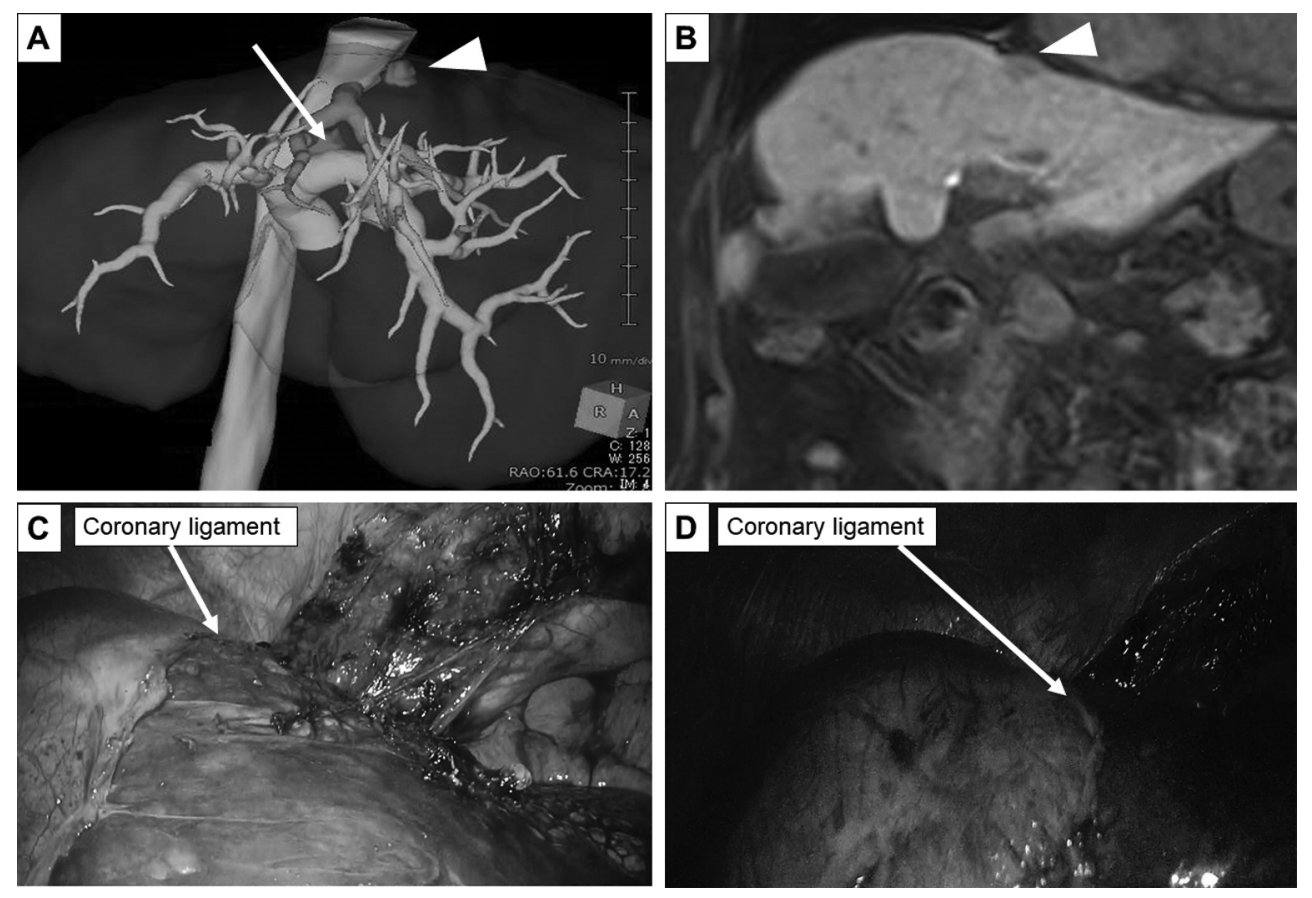

Figure 1. Imaging and intraoperative findings of Patient \#1. (A) Tumours located in segment 1 (arrow) and 4 (arrowhead) on three-dimensional reconstruction. (B) Hepatic phase in axial gadolinium ethoxybenzyl diethylenetriamine pentaacetic acid-enhanced magnetic resonance imaging. During the operation, the tumour in segment 4 was not detected using $(C)$ laparoscopic observation or $(D)$ indocyanine green fluorescence imaging.
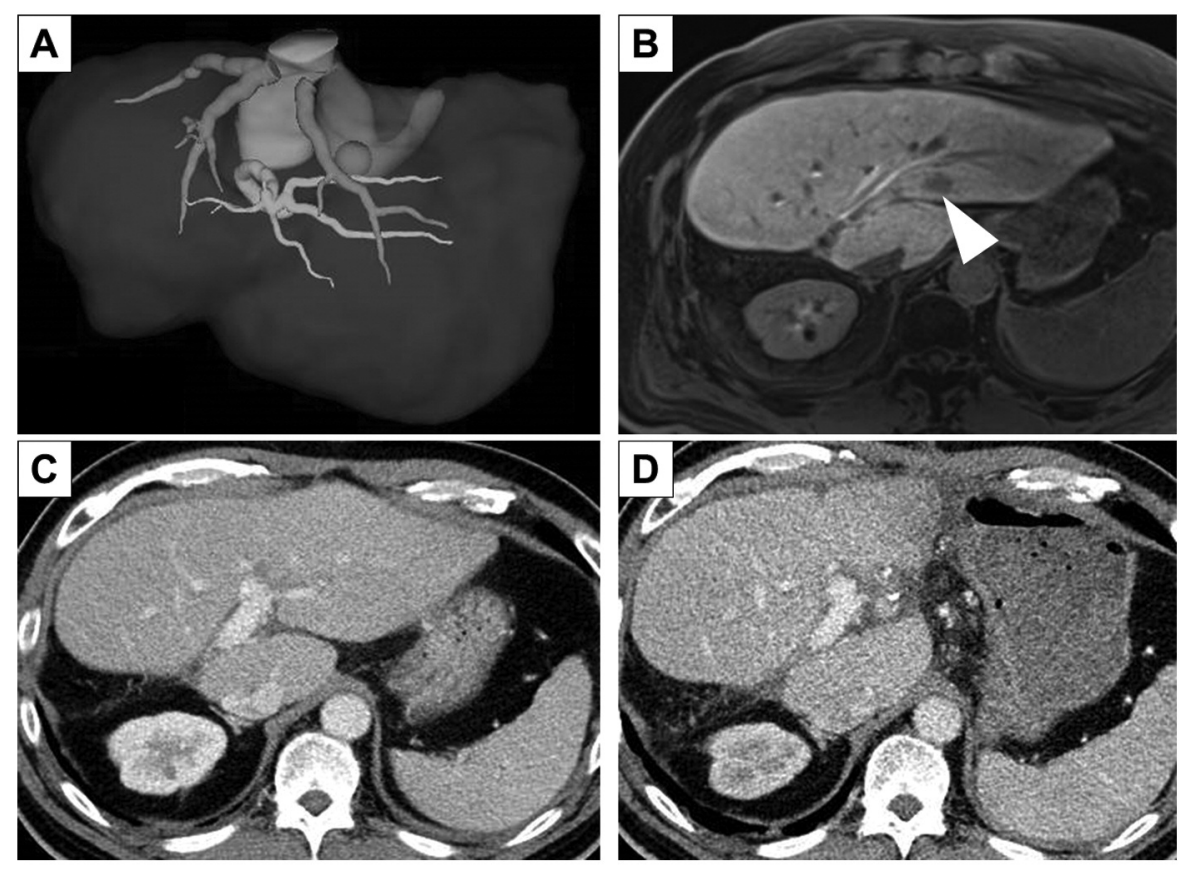

Figure 2. Imaging findings of Patient \#5. (A) Tumour located in segment 2 on three-dimensional reconstruction. (B) Hepatic phase in gadolinium ethoxybenzyl diethylenetriamine pentaacetic acid-enhanced magnetic resonance imaging (white arrowhead). (C) The Glissonean branch of segment 2-oriented partial hepatectomy and postoperative dynamic computed tomography revealed that the Glissonean branch of segment 2 was misrecognized as the Glissonean branch of segment 3. (D) Segment 2 segmentectomy was performed and postoperative dynamic computed tomography showed curative resection. G2: Glissonean branch of segment 2; LHV: left hepatic vein. 
detecting deeply located cancers, however, intraoperative ICG fluorescence imaging is still useful because it not only aids visual inspections but also compensates for drawbacks in IOUS when observing small lesions located immediately beneath the liver surface.

All tumours in the present study were enhanced in the arterial phase and rapidly washed out in the venous phase during dynamic CT and Gd-EOB-MRI, which is consistent with the features of classical HCC. Indeed, four of six tumours were HCCs, and all of these were well-differentiated HCCs. However, even tumours on the surface were not detected using normal light and ICG fluorescence imaging.

Hepatectomy for non-hypervascular nodules does not improve the prognosis, and such patients should be carefully followed up (13); however, all the tumours in our study were hyper-vascular HCCs. Therefore, a new method is needed for resection of these invisible tumours. We usually perform $\mathrm{LH}$ for invisible tumours based on anatomical landmarks including the hepatic vein, Glissonean pedicle, and cut surface of the previous hepatectomy. According to our previous study (14), tumours in segment 3 can be clearly detected using IOUS, whereas tumours in segment 2 are difficult to detect using IOUS. Thus, invisible tumours in segment 2 are a good indication for anatomical resection, including segmentectomy or left lateral sectionectomy. Notably, however, landmarks can be misidentified. In several institutions, the CT-guided hookwire marking technique (15) and three-dimensional printing (16) are currently applied to localize tumours.

Several limitations must be considered when interpreting the present findings. The study was conducted in a single institution with a small sample size. In addition, the IOUS techniques varied among the surgeons. Our findings should be confirmed in larger prospective studies or even a multiinstitutional retrospective study.

In conclusion, we have herein presented a case series of LH for intraoperative invisible tumours. Small tumours in the cirrhosis milieu are poorly visible using IOUS and ICG fluorescence imaging. It is important to note that invisible tumours using POUS may not be identified intraoperatively during LH. For invisible tumours on IOUS, LH based on anatomical landmarks should be preferred.

\section{Conflicts of Interest}

The Authors have no conflicts of interest to declare in relation to this study.

\section{Authors' Contributions}

Masashi Tsunematsu: Study design, data collection and analysis, and manuscript drafting. Shinji Onda: Data collection and manuscript revision. Tomohiko Taniai: Data collection. Mitsuru Yanagaki: Data collection. Koichiro Haruki: Data collection. Yoshihiro Shirai: Data collection. Jungo Yasuda: Data collection. Kenei Furukawa: Data collection. Taro Sakamoto: Data collection. Takeshi Gocho: Data collection. Hiroaki Shiba: Data collection. Toru Ikegami: Manuscript revision and final approval.

\section{Acknowledgements}

This study was supported by JSPS KAKENHI Grant Number 21K15515. We thank Angela Morben, DVM, ELS, from Edanz (https://jp.edanz.com/ac) for editing a draft of this manuscript.

\section{References}

1 Wakabayashi G, Cherqui D, Geller DA, Buell JF, Kaneko H, Han HS, Asbun H, O'Rourke N, Tanabe M, Koffron AJ, Tsung A, Soubrane O, Machado MA, Gayet B, Troisi RI, Pessaux P, Van Dam RM, Scatton O, Abu Hilal M, Belli G, Kwon CH, Edwin B, Choi GH, Aldrighetti LA, Cai X, Cleary S, Chen KH, Schön MR, Sugioka A, Tang CN, Herman P, Pekolj J, Chen XP, Dagher I, Jarnagin W, Yamamoto M, Strong R, Jagannath P, Lo CM, Clavien PA, Kokudo N, Barkun J and Strasberg SM: Recommendations for laparoscopic liver resection: a report from the second international consensus conference held in Morioka. Ann Surg 261(4): 619-629, 2015. PMID: 25742461. DOI: 10.1097/SLA.0000000000001184

2 Yoneyama M, Takayama Y, Nishie A, Nakamura M, Katsumata Y, Takemura A, Obara M, Okuaki T, Honda H and Van Cauteren M: Differentiation of hypointense nodules on gadoxetic acidenhanced hepatobiliary-phase MRI using $\mathrm{T}_{2}$ enhanced spin-echo imaging with the time-reversed gradient echo sequence: An initial experience. Eur J Radiol 95: 325-331, 2017. PMID: 28987688. DOI: 10.1016/j.ejrad.2017.08.025

3 Bismuth H, Castaing D and Garden O: The use of operative ultrasound in surgery of primary liver tumors. World Journal of Surgery 11(5): 610-614, 2019. DOI: 10.1007/BF01655836

4 Viganò L, Ferrero A, Amisano M, Russolillo N and Capussotti L: Comparison of laparoscopic and open intraoperative ultrasonography for staging liver tumours. Br J Surg 100(4): 535-542, 2013. PMID: 23339035. DOI: 10.1002/bjs.9025

5 Lee MW, Kim YJ, Park HS, Yu NC, Jung SI, Ko SY and Jeon HJ: Targeted sonography for small hepatocellular carcinoma discovered by CT or MRI: factors affecting sonographic detection. AJR Am J Roentgenol 194(5): W396-W400, 2010. PMID: 20410384. DOI: 10.2214/AJR.09.3171

6 Pan F, Do TD, Vollherbst DF, Pereira PL, Richter GM, Faerber M, Weiss KH, Mehrabi A, Kauczor HU and Sommer CM: Percutaneous irreversible electroporation for treatment of small hepatocellular carcinoma invisible on unenhanced CT: A novel combined strategy with prior transarterial tumor marking. Cancers (Basel) 13(9): 2021, 2021. PMID: 33922067. DOI: 10.3390/cancers13092021

7 Abo T, Nanashima A, Tobinaga S, Hidaka S, Taura N, Takagi K, Arai J, Miyaaki H, Shibata H and Nagayasu T: Usefulness of intraoperative diagnosis of hepatic tumors located at the liver surface and hepatic segmental visualization using indocyanine green-photodynamic eye imaging. Eur J Surg Oncol 41(2): 257264, 2015. PMID: 25447030. DOI: 10.1016/j.ejso.2014.09.008

8 Viganò L, Ferrero A, Amisano M, Russolillo N and Capussotti L: Comparison of laparoscopic and open intraoperative ultrasonography for staging liver tumours. Br J Surg 100(4): 535-542, 2013. PMID: 23339035. DOI: 10.1002/bjs.9025 
9 Terasawa M, Ishizawa T, Mise Y, Inoue Y, Ito H, Takahashi Y and Saiura A: Applications of fusion-fluorescence imaging using indocyanine green in laparoscopic hepatectomy. Surg Endosc 31(12): 5111-5118, 2017. PMID: 28455774. DOI: 10.1007/ s00464-017-5576-z

10 Kaibori M, Matsui K, Ishizaki M, Iida H, Sakaguchi T, Tsuda T, Okumura T, Inoue K, Shimada S, Ohtsubo S, Kusano M, Ikehara Y, Ozeki E, Kitawaki T and Kon M: Evaluation of fluorescence imaging with indocyanine green in hepatocellular carcinoma. Cancer Imaging 16: 6, 2016. PMID: 27052371. DOI: 10.1186/s40644-016-0064-6

11 Ishizawa T, Fukushima N, Shibahara J, Masuda K, Tamura S, Aoki T, Hasegawa K, Beck Y, Fukayama M and Kokudo N: Real-time identification of liver cancers by using indocyanine green fluorescent imaging. Cancer 115(11): 2491-2504, 2009. PMID: 19326450. DOI: $10.1002 /$ cncr.24291

12 Nakaseko Y, Ishizawa T and Saiura A: Fluorescence-guided surgery for liver tumors. J Surg Oncol 118(2): 324-331, 2018. PMID: 30098296. DOI: 10.1002/jso.25128

13 Takeishi K, Yoshizumi T, Itoh S, Yugawa K, Yoshiya S, Toshima T, Harada N, Ikegami T, Nishie A and Mori M: Surgical indications for hepatocellular carcinoma with non-hypervascular hypointense nodules detected by Gd-EOB-DTPA-enhanced MRI. Ann Surg Oncol 27(9): 3344-3353, 2020. PMID: 32246316. DOI: $10.1245 / \mathrm{s} 10434-020-08419-4$
14 Furukawa K, Haruki K, Yasuda J, Onda S, Taniai T, Hamura R, Shiozaki H, Shirai Y, Iida T, Sakamoto T, Gocho T and Ikegami T: Strategies to perform curative laparoscopic repeat hepatectomy for recurrent liver tumors after open right lobectomy. Anticancer Res 41(4): 2171-2175, 2021. PMID: 33813429. DOI: 10.21873/anticanres.14990

15 Monden K, Sadamori H, Hioki M, Nakano K, Asami S, Ohno S, Sasaki K, Ueki T, Yabushita K, Uka M, Hyodo T, Sakaguchi K and Takakura N: Usefulness of a CT-guided hookwire marking in laparoscopic partial hepatectomy for hepatocellular carcinoma invisible on ultrasonography. Asian J Endosc Surg 10(1): 100103, 2017. PMID: 28045236. DOI: 10.1111/ases.12321

16 Igami T, Nakamura Y, Hirose T, Ebata T, Yokoyama Y, Sugawara G, Mizuno T, Mori K and Nagino M: Application of a threedimensional print of a liver in hepatectomy for small tumors invisible by intraoperative ultrasonography: preliminary experience. World J Surg 38(12): 3163-3166, 2014. PMID: 25145821. DOI: $10.1007 / \mathrm{s} 00268-014-2740-7$

Received July 3, 2021

Revised July 20, 2021

Accepted July 21, 2021 